\title{
An analysis of an optical vortices interferometer with focused beam
}

\author{
EwA FRĄCZEK ${ }^{1 *}$, GRZEGORZ BUDZYŃ ${ }^{2}$ \\ ${ }^{1}$ Chair of Electronic and Photonic Metrology, Wrocław University of Technology, \\ ul. B. Prusa 53/55, 50-317 Wrocław, Poland \\ ${ }^{2}$ Institute of Telecommunication, Teleinformatics and Acoustics, Wrocław University of Technology, \\ ul. Janiszewskiego 7/9, 50-372 Wrocław, Poland
}

${ }^{*}$ Corresponding author: ewa.fraczek@pwr.wroc.pl

\begin{abstract}
In recent years a lot of effort was put into testing and improving the idea of a three-beam interferometer known as an optical vortices interferometer (OVI). Devices based on the idea of the OVI allow measuring small rotation angles and small shifts with a superb resolution. Unfortunately, there are still many problems that have to be solved before an OVI-based device can go into production. In this paper, theoretical calculations and experimental results of using another type of OVI, OVI with focused beam, are presented. The results of measuring small displacements and small rotation angles of reflecting areas are also shown.
\end{abstract}

Keywords: optical vortex, interferometry.

\section{Introduction}

Optical vortices have been currently widely published. This is because they may be potentially used in many demanding areas. Recently one of the most popular is an optical manipulation of very small particles. The so-called optical tweezers [1-3] are used for displacing particles with the use of either white or monochromatic light. White light based optical vortices can also be used in quantum communication [4].

In other publications, the optical vortices are reported to be used, among others, for checking the flatness of the wavefront with the use of the Gaussian beam transporting an optical vortex [5]; for wavefront reconstruction [4, 6, 7]; for formation of the two-dimensional photonic structures with the use of three elliptically polarized waves [8]. Optical vortices are also used in measurements of microshifts [9-11] and in the lasers based on nonlinear effects [12] where the beam containing optical vortices is used as an external pumping beam [13].

Another potentially interesting field of application of the vortices is a superresolution microscope (SM). The resolution of such a microscope is a fraction of the used 
wavelength. The trials of using phase singularities, especially optical vortices, for building an SM began in the late 80ies of the XX century [14-16].

In recent years the optical vortices became, in the nano-metrology, a well-known measurement tool. One of the methods of using OVs in measurements is tracing the deformation of the regular lattice of vortices. The lattice can be generated, e.g., with the use of a three beam interferometer. Current progress in OVIs [18, 19] increases the probability of using OVIs in superresolution applications.

In this paper, an analysis of the OVI working with focused beam is presented. Such an OVI can be used for measuring small shifts and rotations of reflecting areas and is presumably suitable for being used in the SM.

\section{Block scheme}

The configuration of elements of the OVI working with focused beam is presented in Fig. 1. It consists of five nonpolarizing beam splitters $B S$, two mirrors $M_{1}$ and $M_{2}$, a lens $\mathrm{L}$ and a CCD camera for observation and registering of the obtained interferograms. In comparison with the classic OVI [17], one of the OVI's BS is substituted by the plane mirror $\mathrm{M}_{1}$. This was done for equalization of optical path lengths in the paths $U_{A}$ and $U_{B}$. In the path $U_{C}$ the lens and another plane mirror $\mathrm{M}_{2}$ is placed. Focused $U_{C}$ beam, reflected from the mirror $\mathrm{M}_{2}$ interferes with the $U_{A}$ and $U_{B}$ beams and the result is registered by the CCD camera. Mirror $\mathrm{M}_{2}$ was mounted on a table allowing both shifting and rotating of the mirror.

The alignment of the OVI with focused beam is similar to the alignment of the classic OVI [17]. During alignment, the mirror $\mathrm{M}_{2}$ has to be placed in the focus point of the lens L. In order to properly align the position of the beam splitters and the mirror $\mathrm{M}_{1}$, the three interferograms have to be registered $-U_{A}$ with $U_{B}$ beam, $U_{A}$ with $U_{C}$ beam and $U_{B}$ with $U_{C}$ beam. The positions of beam splitters and mirror $\mathrm{M}_{1}$ have to be altered until the shape of the registered interferograms is proper.

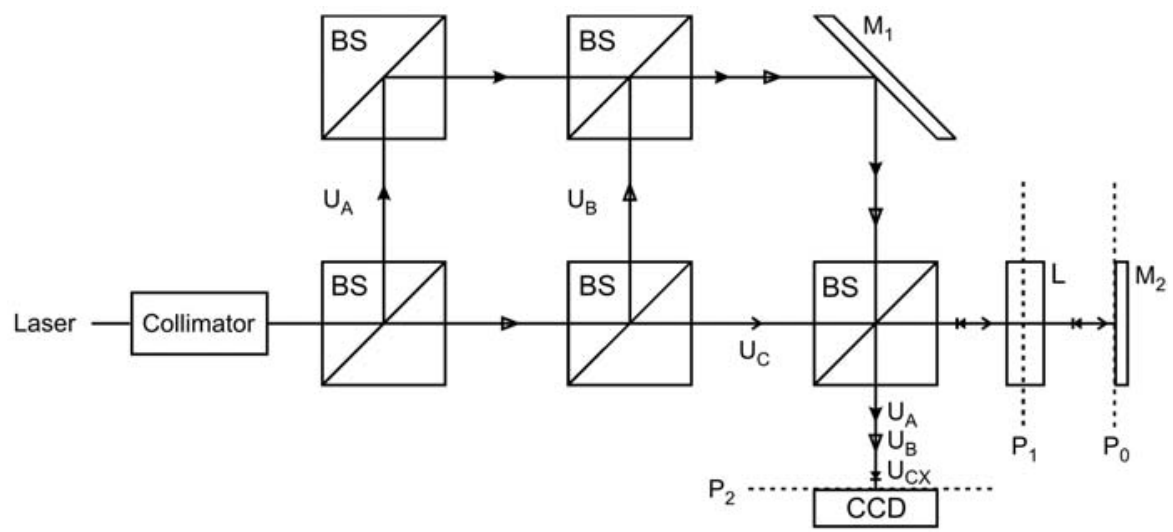

Fig. 1. Block scheme of an OVI working with focused beam; $B S$ - beam splitter; $M_{1}, M_{2}-$ mirrors; $\mathrm{L}$ - lens; CCD - camera; $P_{0}, P_{1}, P_{2}$ - planes. 


\section{Theoretical model}

In the theoretical calculations we focused on the analysis of the $U_{C}$ beam wavefront changes caused by the mirror $\mathrm{M}_{2}$ movement. For this purpose a model was constructed. In this model the plane wave $U_{C}$ passing the aberrational free lens is focused, then reflected by the mirror and then, after a return pass through the lens and redirection on the BS, interferes with the waves $U_{A}$ and $U_{B}$. During theoretical analysis, two cases were taken into consideration: case 1 - beam $U_{C}$ is focused exactly on the plane of the mirror $\mathrm{M}_{2}$; case 2 - mirror plane is placed less than $1 \mathrm{~mm}$ from the focus point of the lens.

\subsection{Calculation procedure}

The image of the incident plane wave transversing an aberration free lens is described as an Airy spot [20]. In the theoretical calculations we made a simplification that the wave is focused to a circularly shaped dot with the diameter of $20 \mu \mathrm{m}$ in the $P_{0}$ plane (i.e., mirror plane - Fig. 1). Changes of the image because of the defocusing of the beam focusing arrangement were modeled by the shape and intensity change of the focused spot. Each point of the spot is treated as a source of a new spherical wave $U_{S}[21]$

$$
U_{S}\left(P_{0}\right)=\frac{A}{r} \exp (i k r)
$$

where $A$ is the amplitude of the wave, $\mathbf{k}$ is the wave vector and $\mathbf{r}$ is the localized vector.

If the mirror $\mathrm{M}_{2}$ is placed outside the focus point of the lens, then the diameter of the dot increases. The size of the dot can be calculated from the aperture of the lens. In the plane of the lens, complex amplitudes of all spherical waves are summing to the wave illuminating the lens $U_{C o}$. In the plane of the lens $P_{1}$ we obtain $(N$ is the number of points in the focused dot)

$$
U_{C o}\left(P_{1}\right)=\sum_{1}^{N} \frac{A_{i}}{r_{i}} \exp \left(i k_{i} r_{i}\right)
$$

After the lens, depending on the mirror $\mathrm{M}_{2}$ position, this wave can be either plane or spherical. The wave is plane only if the mirror $\mathrm{M}_{2}$ is placed in the focus point of the lens. Otherwise the wave is spherical and is converging to one point or scattering. In this point, the geometric picture of all lighting points appears. In order to take into account all the above cases, the superposition rule was used. According to this rule, each solution of the wave equation can be presented as a superposition of monochromatic plane waves with different frequencies, amplitudes, phases and propagation directions.

Because of the above each point reached by the wave $U_{C o}$ becomes the source of the plane wave with the amplitude of the $U_{C o}$ wave and the phase of this wave in 
the point. The direction of the plane wave is calculated from the position of the images of all light source points [21]. Complex amplitudes $U_{C X}$ of these plane waves are summed in the point placed $50 \mathrm{~cm}$ from the mirror $\mathrm{M}_{2}$, i.e., in the place where the interferograms were registered in the real setup (in the plane $P_{2}$ ).

After making all the above described steps, the complex amplitude of the $U_{C x}$ wave is obtained. This wave carries information about the position of the mirror $\mathrm{M}_{2}$. In the last step, the amplitudes of the $U_{A}, U_{B}$ and $U_{C X}$ waves are summed.

\subsection{Results of theoretical calculations}

The results of the numerical calculations will be presented as pictures showing the positions of optical vortices depending on the position of the mirror $\mathrm{M}_{2}$. The charts were calculated in the situation when the mirror was placed outside the focus point of the lens. When the mirror $\mathrm{M}_{2}$ was placed exactly in the focus point there was observed no changes in the position of the optical vortices during rotation of the mirror (that is the reason why, during alignment of the setup, the mirror $\mathrm{M}_{2}$ has to be placed in the focus point of the lens).

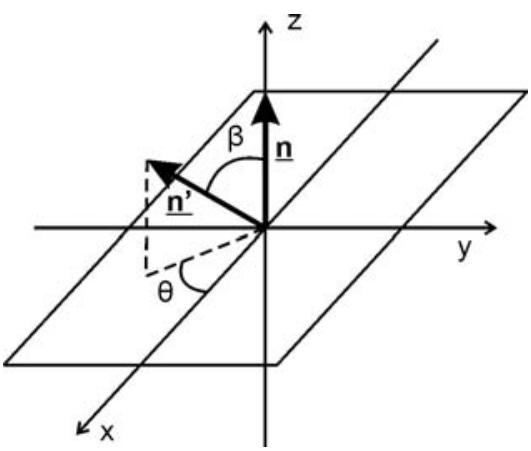

Fig. 2. Angles $\beta$ and $\theta$ describing the rotation of the vector perpendicular to the surface of the mirror.

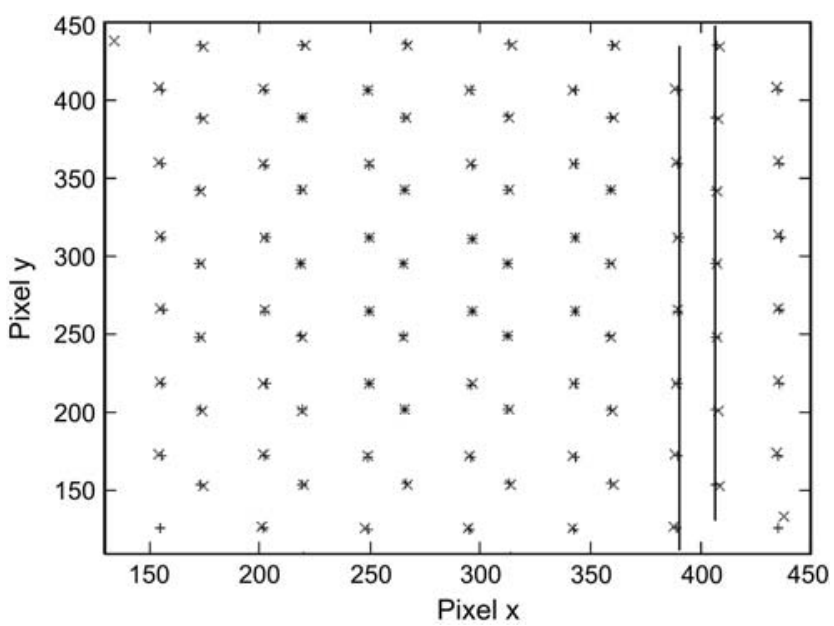

Fig. 3. Net of optical vortices in the case when mirror $\mathrm{M}_{2}$ was moved along $z$-axis without rotation: $z=0.05 \mathrm{~mm}(+), z=0.15 \mathrm{~mm}(\times)$. 
The area and the density of the vortices were smaller than in the experiments in order to reduce the time duration of the calculations. In the calculations, the mirror rotations were described by two angles $\beta$ and $\theta$-see Fig. 2. The values of these angles were chosen so, that they could be feasibly set in the experimental setup. The value of the angle $\beta$ did not exceed 2 degrees.

In Figure 3 the situation in which the angles $\beta$ and $\theta$ were 0 and the mirror $\mathrm{M}_{2}$ was placed $0.15 \mathrm{~mm}$ from the focus point is presented. The mirror was translated by $\Delta z=0.1 \mathrm{~mm}$. It is noticeable that in the central point of the chart the shift of the optical vortices is indiscernible. The further from the center, the larger is the shift. The sym-

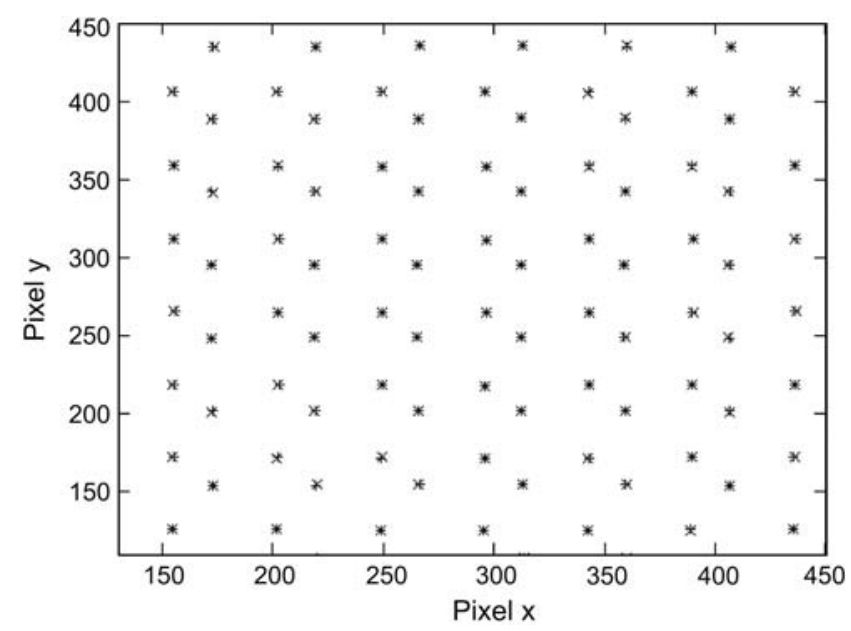

Fig. 4. Net of optical vortices in the case when mirror $\mathrm{M}_{2}$ was rotated at an angle $\beta$ (angle $\theta=0^{\circ}$ ): $\beta=0^{\circ}(+), \beta=0.1^{\circ}(\times)$. Mirror $\mathrm{M}_{2}$ was set in position $z=0.15 \mathrm{~mm}$ before the lens.

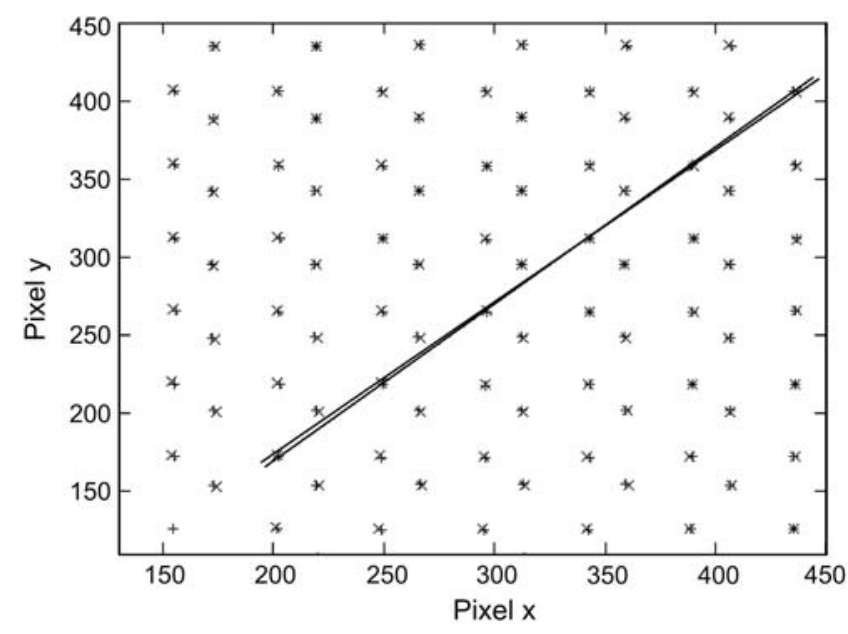

Fig. 5. Net of optical vortices in the case when mirror $\mathrm{M}_{2}$ was rotated by an angle $\beta$ (angle $\theta=45^{\circ}$ ): $\beta=0^{\circ}(+), \beta=0.5^{\circ}(\times)$. Mirror $\mathrm{M}_{2}$ was set in position $z=0.05 \mathrm{~mm}$ before the lens. 
metry of this shift in dependence on the central point is observed. It is so, because the $U_{C X}$ wave is spherical.

In Figure 4 the situation with $\beta=0.1^{\circ}, \theta=0^{\circ}$ and the mirror not moving $(\Delta z=0)$ was presented. Mirror $\mathrm{M}_{2}$ was located $0.15 \mathrm{~mm}$ from the focus point. In the simulated conditions, optical vortices changed their positions - vortices of different signs were moving in different directions but it was difficult to find from the results that the mirror was rotated by a small angle in one axis. In the results presented in Fig. 4 there exists no such symmetry as in the results presented in Fig. 3.

Next picture (Fig. 5) shows the situation when $\beta=0.5^{\circ}, \theta=45^{\circ}$ and the mirror is still $(\Delta z=0)$, placed $0.05 \mathrm{~mm}$ before the focus point. As in the previous example there is no symmetry in the position of vortices. Lack of symmetry results from the still position of the mirror. In Figure 5 points with the same topological charge were connected. It can be noticed that these lines are not parallel. The value of the angle between these lines depends on the value of the $\beta$ angle.

\section{Measurement results}

The measurements were done in the setup presented in Fig. 1. In Figure 6 there is shown one of the pictures registered by the CCD camera during performed measurements. In this picture, the crosses mark the positions of the optical vortices. They were localized with the use of the triangle method [22]. In order to be able to present two lattices of vortices on one picture, we decided to show further (Figs. 7 and 8) only the localized vortices and not the true registered data.

In Figure 7 there were shown results obtained during mirror $\mathrm{M}_{2}$ shift along $z$-axis. The distances between points marked by "+" are, when analyzing on $y$-axis, lower than distances between points marked by " $\times$ ". Such effects were also observed during theoretical calculations (Fig. 3) - during lens' defocusing vortices were changing their positions along radiuses coming from the central point. In the central area, the positions of both registered vortex lattices were the same. In Figure 7 the central point is

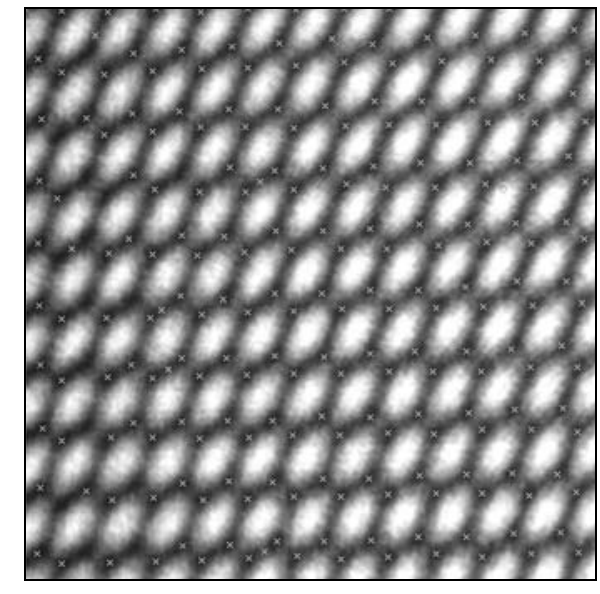

Fig. 6. A picture registered during measurements by the CCD camera. Positions of the optical vortices (crosses) were found with the use of the triangle method [22]. 


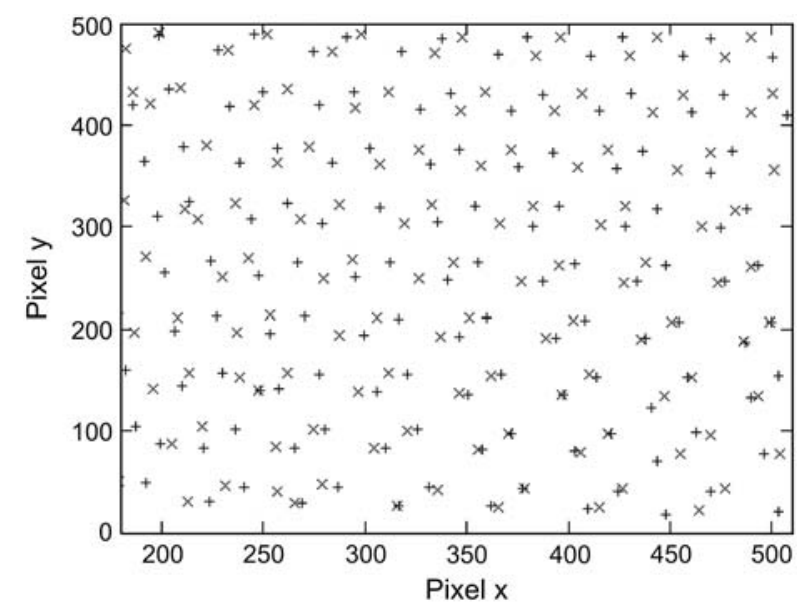

Fig. 7. Measurement results obtained during mirror $M_{2}$ shifting along $z$-axis $(\Delta z=0.3 \mathrm{~mm})$ : mirror set in $\mathrm{Z}=0.05 \mathrm{~mm}$ position $(\times)$, mirror set in $\mathrm{z}=0.45 \mathrm{~mm}$ position $(+)$.

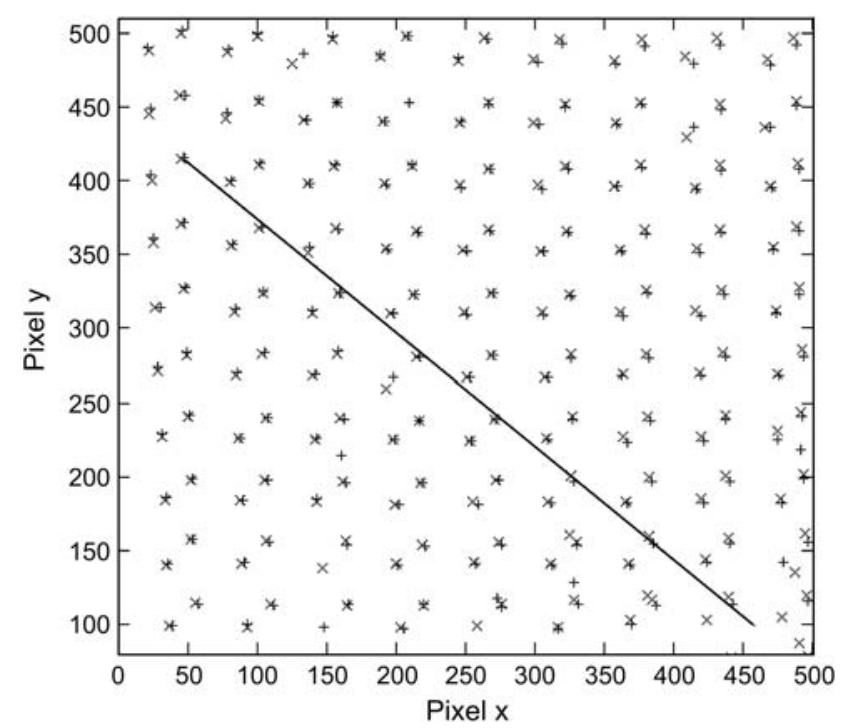

Fig. 8. Measurement results obtained during mirror $\mathrm{M}_{2}$ rotation by an angle $\beta$ (angle $\theta \approx 45^{\circ}$ ): $\beta \approx 0.3^{\circ}(+), \beta \approx 0.6^{\circ}\left(\Delta \beta \approx 0.3^{\circ}\right)(\times)$.

located near the $(370,90)$ point (size of one pixel is $6.45 \times 6.45 \mu \mathrm{m}$ ). The analysis of the vortices marked by "+" shows that the mirror was not only shifted but also rotated. The observed rotation was not intended. It was a side effect of the movement of the used table.

Figure 8 illustrates the case when the mirror was not shifted but rotated. Despite the wavefront distortion of the $U_{C X}$ wave that is influencing the regularity of the vortex lattice, it can be discerned that the shifts of the points have the same character like 
the one presented in Fig. 5. In Figure 8 vortices marked by "+" and with identical topological charge were connected with the line. Optical vortices marked by " $\times$ " are, in the upper part of the interferogram, placed on the left side of the line.

The changes in the location of the optical vortices that were observed during measurements have the same character like the changes obtained in numerical calculations.

\section{Conclusions}

From the presented theoretical calculations it arises that in the position changes of the optical vortices there is hidden information about the movement of the mirror $\mathrm{M}_{2}$. It is important to notice that the position of the mirror in relevance to the focus point of the lens influences the easiness of the analysis of the vortices picture.

Moreover, the distance from the mirror to the focus point influences the wavefront shape of the reflected wave. The larger is this distance, the larger is the curvature of the wavefront. The increase of the curvature is not desired, so the measured objects should not be placed further than $0.1 \mathrm{~mm}$ from the focus point of the lens. The vertical measurement range is approx. $100 \mu \mathrm{m}$.

Because of the current testing procedure it cannot be yet foreseen how will the edges and rifts of the examined surface influence the position of the vortices. Such analyses are planned in the future.

In general, the experimental data comply with the data obtained in numerical calculations. During measurements a lot of troubles was caused by the quality of the optical (splitters and lens) and mechanical components used in the setup. In the proposed setup, the quality of the used elements severely influences the quality of the recorded interferograms. In the next stages of the researches we intend to modify the setup to get more accurate results.

On the current state of the research it can be stated that in the described setup it is possible to measure small rotation angles of a small surface. The size of this surface should not exceed $50 \times 50 \mu \mathrm{m}$. The range of the measurable angles, obtained in the numerical calculations, is from 10 arcsec to 2 degrees.

Acknowledgments - We acknowledge the support of this work by the Foundation for Polish Science.

\section{References}

[1] Allen L., Barnett S.M., Padgett M.J., Optical Angular Momentum, Institute of Physics Publishing, London 2003.

[2] Ashrin A., Dziedzic J.M., Buorkholm J.E., Chu S., Observation of a single-beam gradient force optical trap for dielectric particles, Optics Letters 11(5), 1986, pp. 288-290.

[3] Grier D.G., A revolution in optical manipulation, Nature 424(6950), 2003, pp. 810-816.

[4] Aksenov V., Banakn V., Tiknomirova O., Potential and vortex features of optical specle fields and visualization of wave-front singularities, Applied Optics 37(21), 1998, pp. 4536-4540.

[5] Senthilkumaran P., Optical phase singularities in detection of laser beam collimation, Applied Optics 42(31), 2003, pp. 6314-6320. 
[6] Kolenovic E., Correlation between intensity and phase in monochromatic light, Journal of the Optical Society of America A 22(5), 2005, pp. 899-906.

[7] Fraczek W., Fraczek E., Mroczka J., High precision parallel glass plate test with the use of the optical vortex interferometer, Proceedings of the SPIE 6189, 2006, p. 618925.

[8] Weidong Mao, Yongchun Zhong, Jianwen Dong, Hezhou Wang, Crystallography of two-dimensional photonic lattices formed by holography of three noncoplanar beams, Journal of the Optical Society of America B 22(5), 2005, pp. 1085-1091.

[9] Wang W., Ishi N., Hanson S.G., Miyamoto Y., Takeda M., Phase singularities in analytic signal of white-light speckle pattern with application to micro-displacement measurement, Optics Communications 248(1-3), 2005, pp. 59-68.

[10] Wang W., Hanson S.G., Miyamoto Y., Takeda M., Experimental investigation of local properties and statistics of optical vortices in random wave fields, Physical Review Letters 94(10), 2005, p. 103902.

[11] Wang W., Yokozeki T., Ishijima R., Wada A., Miyamoto Y., Takeda M., Hanson S.G., Optical vortex metrology for nanometric speckle displacement measurement, Optics Express 14(1), 2006, pp. $120-127$.

[12] Desyatnikov A.S., Torner L., Kivshar Yu.S., Optical vortices and vortex solitons, [In] Progress in Optics, [Ed.] E. Wolf, Vol. 47, 2005.

[13] Oppo G.-L., Scroggie A.J., Firth W.J., Characterization, dynamics and stabilization of diffractive domain walls and dark ring cavity solitons in parametric oscillators, Physical Review E 63(6), 2001, p. 066209.

[14] Tychinsky V.P., On superresolution of phase objects, Optics Communications 74(1-2), 1989, pp. 41-45.

[15] Tychynsky V.P., Velzel C.H.F., Super-resolution in microscopy, [In] Current Trends in Optics, Chapter 18, Academic Press, 1994.

[16] Velzel C., Masajada J., Superresolution phase image microscope, Optica Applicata 29(3), 1999, pp. 293-300.

[17] Masajada J., Dubik B., Optical vortex generation by three plane wave interference, Optics Communications 198(1-3), 2001, pp. 21-27.

[18] Masajada J., Small-angle rotations measurement using optical vortex interferometer, Optics Communications 239(4-6), 2004, pp. 373-381.

[19] Popiolek-Masajada A., Borwińska M., Frączek W., Testing a new method for small-angle rotation measurements with the optical vortex interferometer, Measurement Science and Technology 17(4), 2006, pp. 653-658.

[20] Stamnes J.J., Waves in Focal Regions, Adam Hilger Series on Optics and Optoelectronics, Bristol and Boston, 1986.

[21] Gniadek K., Optyczne przetwarzanie informacji, PWN, Warszawa 1992 (in Polish).

[22] Masajada J., Popiolek-Masajada A., Frączek E., Frączek W., Vortex points localization problem in optical vortices interferometry, Optics Communications 234(1-6), 2004, pp. 23-28. 